\title{
A Critical Approach for Evolution Analysis in Regenerative Developments
}

\section{Ali M S Kashkooli ${ }^{1 *}$, Ehsan Daneshi ${ }^{2}$ and Abraham M Hashemian ${ }^{2}$}

${ }^{1} A K I D$ Consultants Ltd., Vancouver, BC, Canada

${ }^{2}$ Simon Fraser University, Vancouver, BC, Canada

\begin{abstract}
Over the recent five years, the Regenerative Design concept has considerably intended to understand the factors of recovering the environmental and socioeconomic damages of the last-century global industrialization. Moreover, it has attempted to consider the evolutions within and out-with its assessing system/s.

As a short communication, this paper attempts to argue the fundamentals to develop precise computerized Evolution Analysis Systems (EAS), in order to address the future identification requirements, of Regenerative Developments.

Therefore, as an interdisciplinary discussion, the paper argues the applicable systems to develop evolutionary algorithms, with a specific focus in Genetic Algorithms (GA), as one of the most efficient approaches to for evolutionary quantification. Furthermore, the paper introduces the Second Life approach as a significant example of evolutionary computation, to achieve better EASs, as a foundation of reliable Regenerative Analysis Systems (RAS).
\end{abstract}

Indeed, the paper suggests, a critical evolution-based Regenerative Analysis approach, as a basis to develop future Regenerative Analysis and Regenerative Design software.

Keywords: Regenerative design; Future identification; Evolutionary algorithms; Evolutionary computation

\section{Introduction- Regenerative Developments}

During the last century the global of industrialisation has caused some un-wanted negative impacts on human socio-economic behaviours as well as the global environment. Although The 'Sustainability Concept' (also introduced as 'green design' concept in many literatures) has tried to address the resource consumptions (such as energy, water, food etc.) and preventation of the further socio-economic and environmental impacts, the previous onecentury damages are still existing. This in turn, has translated into a further solution recently introduced as 'Regeneration' or 'Regenerative Developments' (RD) $[1,2]$. The main challenge of $\mathrm{RD}$ is to provide and to consider the potentials for 'Renew', 'Re-birth' and 'recover' the previously caused damages to human socio-economic relations as well as the environment. Accordingly, the regeneration concept intends on further than 'generation-consumption' systems (Figure 1). Therefore, in order to be regenerative, a system must provide the factors such as 'being sustainable for today', 'being sustainable for tomorrow', and 'recovering the previously caused damages on economy, society and environment'.

Therefore, a set of "resource-related strategies within cycles - from nature and back to nature" have been proposed as follows:

- "Produce: resources are renewable and are sourced or generated either onsite or locally.

- Use: resources are used effectively in satisfying human needs.

- Recycle: resources are used for multiple purposes and benefits.

Replenish: rather than diminish natural capital during the production of resources and assimilation of 'waste', replenishes and builds natural capital." $[4,5]$.

Regenerative Design considers a much more complex set of potential exchanges associated with both Net Zero and Net Positive systems. Accordingly, the impacts are being assessed in the following ways [5]

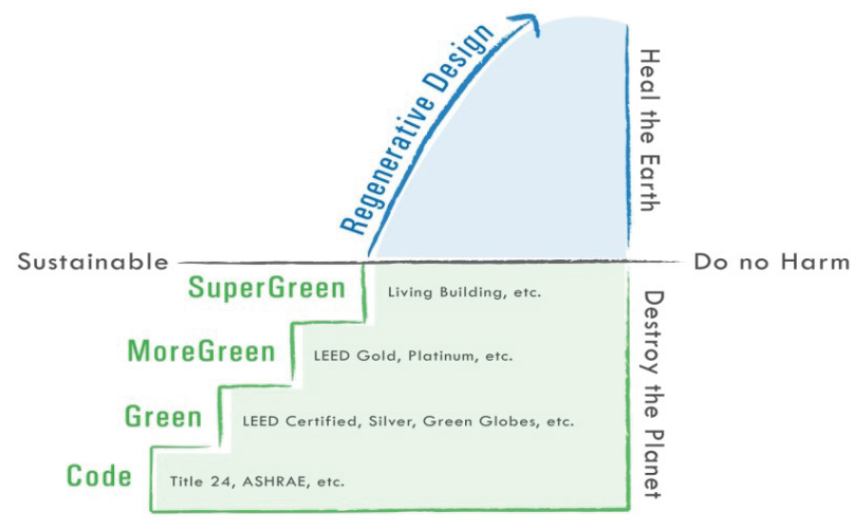

Figure 1: Regenerative vs. Sustainable; the general concept [3].

Grid-connected impacts: includes all inter-connections of the system/s with their surrounding system/s and neighbourhoods and exchanges of energy, water, wastes etc. "Grid connection is a necessary and core requirement of net zero/[positive] energy buildings" [5].

- Waste scavenging: includes spotting the wastes and problems within the existing systems and infrastructures to prevent further damages. This process considers the improvements of

*Corresponding author: Ali M S Kashkooli, Principal of AKID Consultants Ltd., \#3002, 1033 Marina side Crescent, Vancouver, BC, Canada, Tel: +1-604-655-3940; E-mail: a.kashkooli@akidltd.com

Received August 13, 2015; Accepted September 01, 2015; Published September 06, 2015

Citation: Kashkooli AMS, Daneshi E, Hashemian AM (2015) A Critical Approach for Evolution Analysis in Regenerative Developments. Int J Swarm Intel Evol Comput 4: 119. doi: 10.4172/2090-4908.1000119

Copyright: (C) 2015 Kashkooli AMS, et al. This is an open-access article distributed under the terms of the Creative Commons Attribution License, which permits unrestricted use, distribution, and reproduction in any medium, provided the original author and source are credited. 
Citation: Kashkooli AMS, Daneshi E, Hashemian AM (2015) A Critical Approach for Evolution Analysis in Regenerative Developments. Int J Swarm Intel Evol Comput 4: 119. doi: 10.4172/2090-4908.1000119

'quality of performance' within the system/s exchanges and the 'quality of Net expectation'.

- Recovery potentials: includes adding of potentials and new infrastructures as well as improvement of the existing systems and/or infrastructures to achieve a faster recovery of the previously caused damage to the assessing society/ies, environment and economic system.

The optimization factors of RD are as follows:

- Covering all requirements of a sustainable system such as economic, social and environmental preservation;

- Considering and providing of infrastructures to address the future requirements of systems, based on future identification;

- Recovering of the existing omissions in-with the systems, caused in past events.

In contrast with Sustainable Developments, the Regenerative system considers the human and environmental activities as interrelated flows', and the impact of each flow on the others in a greater scale (Figure 2).

\section{Role of Evolution and Future Identification}

As the Regenerative Design intends to provide a better system which recognises human as a part of the 'ecosystem'; and respects to the need of human to be incorporated into it. This in turn, is being translated into another model for sustainable living "that relies on synergy, or the idea of separate components forming a whole that is greater than the sum of its parts. It emphasizes patterns and groupings that occur naturally"[7].

Accordingly, the Regenerative Design attempts to consider the evolutions within and out-with its assessing system/s. "[t]he All these ideas combine to create patterns that mimic nature so that humans can take a symbiotic role in their environment rather than a destructive one. Obviously, a perfect closed system that regenerates itself 100 per cent is not mathematically possible, so the current goal is 99.9 per cent regeneration. Even that goal is a challenging one, but the process of attempting it is viable, and the result of not adapting to a changing environment is very clearly demonstrated in history: it is extinction" [7] (Figure 3).

As previously stated, one of the main attributions of regenerative

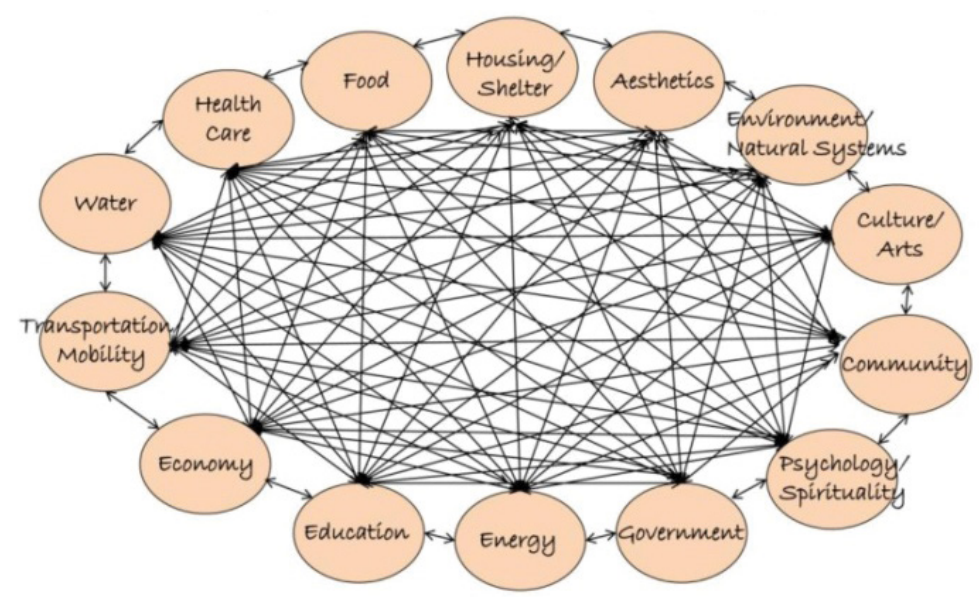

Figure 2: The inter-relation of the flows in a Regenerative Community [6].

Regenerating system

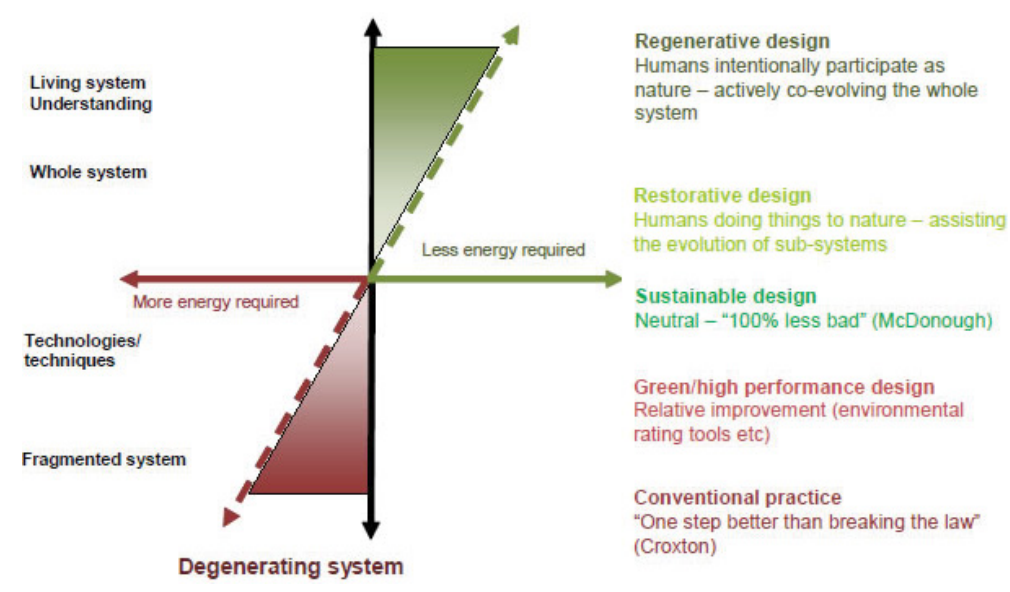

Figure 3: The evolution-based view of RD [8]. 
design is to have a 'net positive' system, which considers in 'providing potentials to deal with future changes' and 'to recover the previously caused damages to environment, society and economy. This in turn, identifies the great demand for 'anticipation and pre-assessment' of the future events. This demand principally comes from the need of market to secure the investments on extra infrastructure for dealing with possible future changes and events. However, the role of uncertainty is supposed to be considered.

In comparison with the length of human life, the systems contain very long-term service-lives. As shown in Figure 4 a typical building neighbourhood (as a system example) can face several changes during its service-life. These changes can be greater and more diverse regarding the longer proposed building timeframes.

The aforementioned changes can be considered in following factors/flows: Population, building occupation and functional changes, end-users' behaviours, ethnics and culture, environmental changes (such as temperature, rain and snow, wind, green area type, lakes, rivers etc.), technology changes (utilities' technology, in-site and out-site energy generation system/s, wireless systems etc.), market changes etc. [9-10]. Moreover, the new developments in a neighbourhood/system will change the traffic and energy-water-waste management systems. Furthermore, the level of pollutions and time-based strategies of pollution management will be altered.

\section{Regenerative Evolutionary Strategies}

The development of human-environment interaction has been known as a complex issue, including lots of difficulties to study, through using of only descriptive verbal languages. Hence, due to the non-linear relationship between different components of the humans' surrounding environment, and to make correct decisions to prevent the global resource depletion, development of a tool to systematically study and to predict the effects of changes in these components is crucial. Evolutionary Computation (EC) [12] is a tool to examine the future options of change, in an artificial universe, established on the historical facts and the evolutionary algorithms, which are developed on those bases. At this stage, the paper investigates the EC methods (with a specific intention in genetic algorithms and artificial life), the fundamental ideas behind them, and their limitations. To continue, the paper discusses the 'Second Life project' as an example of evolutionary virtual environment, in order to highlight the process of software development for EASs.

EC is one of the well-known fields of Artificial Intelligence (AI), which employs the evolved biological mechanisms for optimisation purposes. Over the last three decades, several method of EC have been introduced such as ant colony [13], genetic algorithm (GE) [14], bees algorithm [15], cuckoo search [16] etc. Along with analytical methods and models, these tools let us study the possible outcomes of large number of scenarios using computers which may significantly reduce the planning and designing cost and time. Genetic algorithm is well known because of its ability in optimizing highly sophisticated models when multi factors need to be optimized simultaneously [17]. This aspect is highly approachable to cover one of the principal targets of $\mathrm{RD}$, which is future identification (Refer to section 1).

\section{Individual representation and objective function}

Established on level of complexity, parameters of interest, and number of optimization factors in-with each system, detailed mathematical models are being developed, which describe the components' interactions, properties of the environment and the goals that one wants to achieve. These goals may include; minimizing the cost, generating the least possible pollution, maximizing the number of housings in a limited urban area and etc. Adapted from Darwin Evolution Theory, each component of the model is presented by a set of binary strings, which are called as 'Chromosomes'. These chromosomes are consisting of genes which individually set their own properties. On the basis of each system goal, a cost function is being defined, which assigns a non-negative value to a proposed set of components (and their properties), as a foundation to achieve a unique solution. This number is called Fitness. In GA, it is generally assumed that the solutions that have better ability to adapt and optimise the cost function have less fitness values. Therefore, the overall goal of GA is to find the best solutions by generating and choosing chromosomes that have minimum fitness values.

Genetic algorithm is a great tool for finding the fittest solution while the solution-space is constrained by several factors $[14,18]$. These constrains may be induced by natural factors such as the amount of available energy, land, temperature and etc. or set by the key decision makers which may include tax rate, budget and etc. It is possible to generate an arbitrary or random set of chromosomes as the first generation of proposed solutions. The fitness values is calculated for each of these solutions by GA and the ones with the lower fitness values are selected to form the next generation of chromosomes which are known as 'Off springs'.

\section{Evolution operators}

In such cases, the crossover and gene mutation are considered as evolution operators, amongst several operators, to be used to combine parents' chromosomes. Each offspring, which is a potential solution for the problem, inherits some of the properties of its previous generation (Also known as its 'Parents'). However, mutations might change offspring's properties significantly. This process maximises the chance to improve the off springs' fitness values, while the defined constrains are not violated. GA continues repeating this process for a certain number of generations or stops when the fitness value by one of the solutions is less than an acceptable value. Both of these stopping criteria are being defined according to the specific demands of the decisions makers/RD planner (Figure 5).
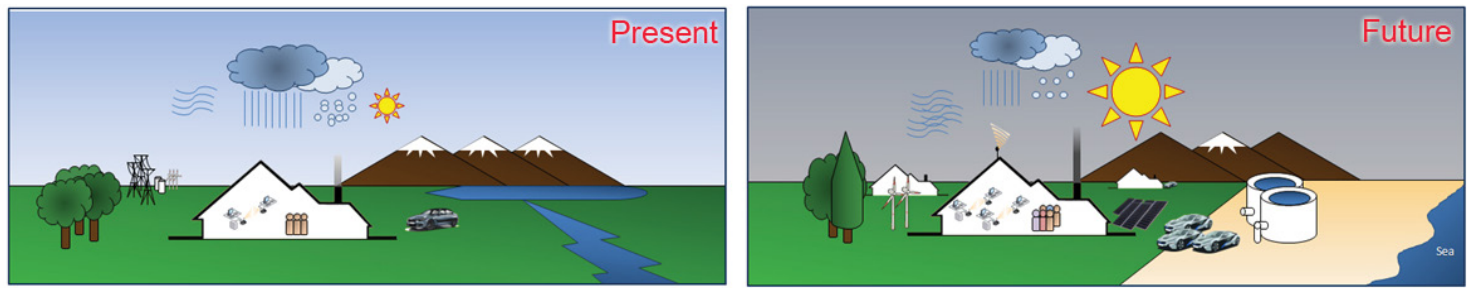

Figure 4: A comparison of internal and external influences on a building in a typical timeframe [11]. 


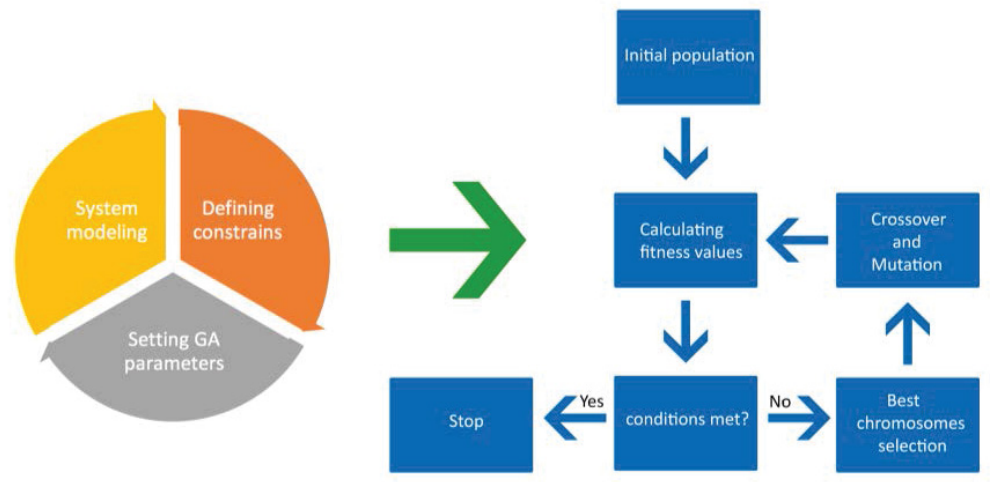

Figure 5: Modelling and optimization using genetic algorithm.

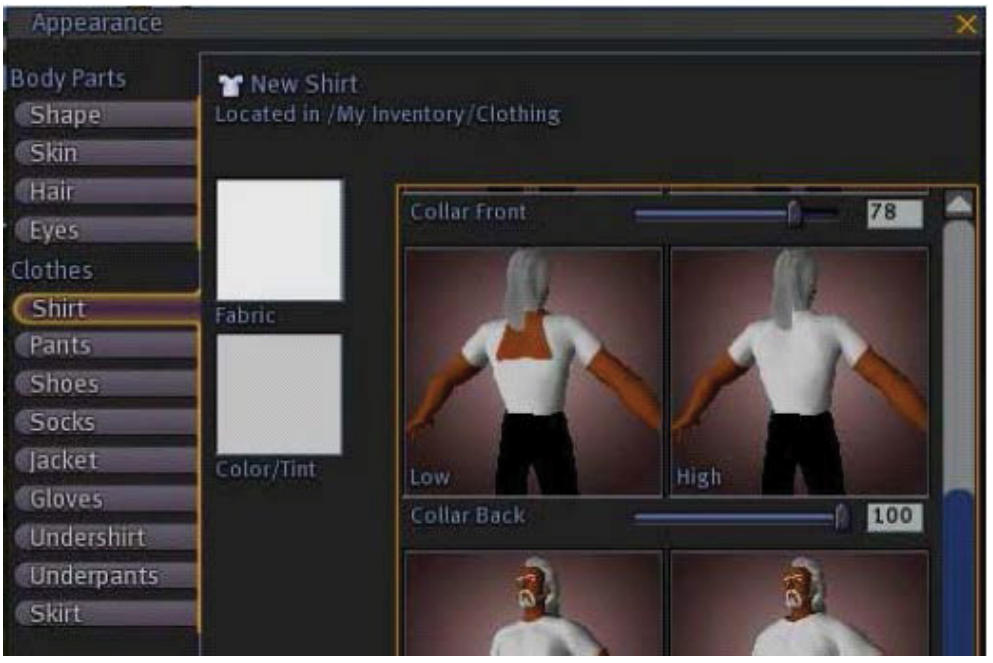

Figure 6: Character creation interface in Second Life [20].

\section{Virtual Simulations of Natural Environments}

In 2003, the Second Life (SL) project has been launched by Linden Research Incorporation as a dedicated Virtual Environments (VE) for human social interactions. Comparing to other VEs, SL has been assumed as an evolution, because unlike other games, people don't have any mission to complete in this environment. As a part of the SL, people spent time to go through this environment (for free) to explore it with their own virtual bodies, called as 'Avatar', which could be designed like (or unlike) their physical bodies (Figure 6). According to the Linden Lab report [19], in 2014, around 1 million users visited there monthly. They spent most of their time for walking, shopping (virtual goods such as dresses etc.), talking with others, making new friends and hanging out with them in virtual places (such as clubs, parks etc.)

There are also other interesting usages to turn virtual environments (such as SL) into a well-used environment for human future interactions. For example, IBM's Academy of Technology hold its annual conference, Academy General Meeting (AGM) through SL to help the attendees to take part of this conference from all over the Earth (Figure 7) [21]. The conference was successful, and $66 \%$ of its 444 participants reported that they had a good experience. In each day, about 350 people attended in keynote events, and between keynotes, people were gathered at social places to form small groups and talk to each other.
In order to provide dynamic simulation possibilities in SL, Linden Lab created LSL (Linden Scripting Language) for its users, so they can change environmental parameters and simulate different types of phenomena in their personal island. Because of its potentials, SL became to an interesting platform to study real life phenomena through virtual simulation. For example, at 2007, University of Delaware (UD) bought its island inside SL and started to build virtual departments (Figure 8). To have a better understanding of SL potentials in terms of simulating pollutants, physic concepts and landscape design, we mention some areas of UD's island, as Debbie Jeffers describe at [22]:

- Art conservation - A building is being set up as a restoration lab, where students and visitors will the see the effects of pollutants on works of art.

- Astronomy and physics - We have an observatory complete with scripted telescope. We will include the latest images from UD space research in it as well as areas for presentations and other educational materials. A graduate student has taken on a project to create some scripted learning modules for some of the more complex physics concepts.

- Landscape design - This department is planning to use the Island for distance education, enhancing the learning experience with virtual physical representations of their landscape drawings. 


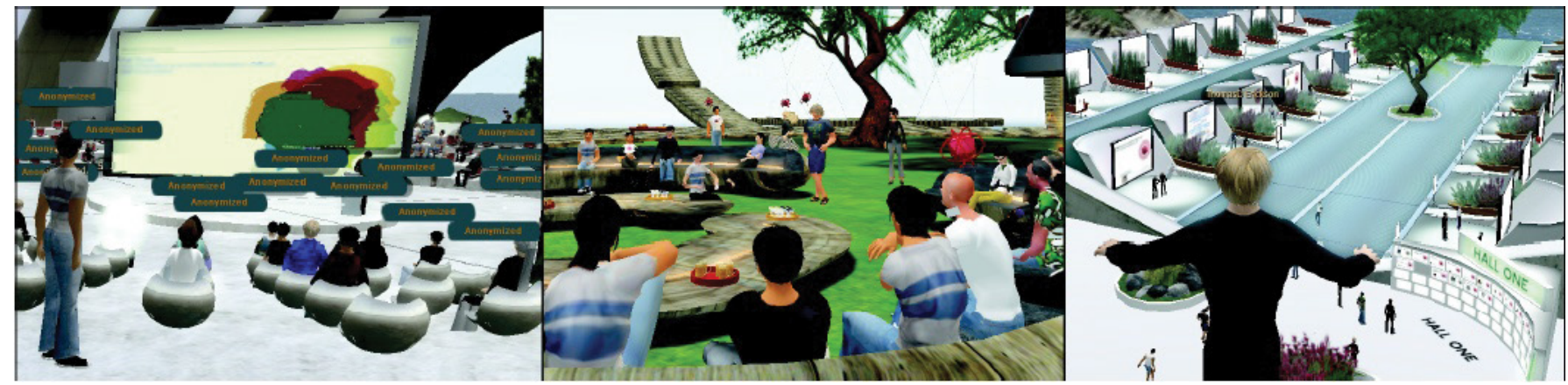

Figure 7: IBM's virtual Conference - Left: A Keynote, Middle: A social Event, Right: A Poster Session [21]

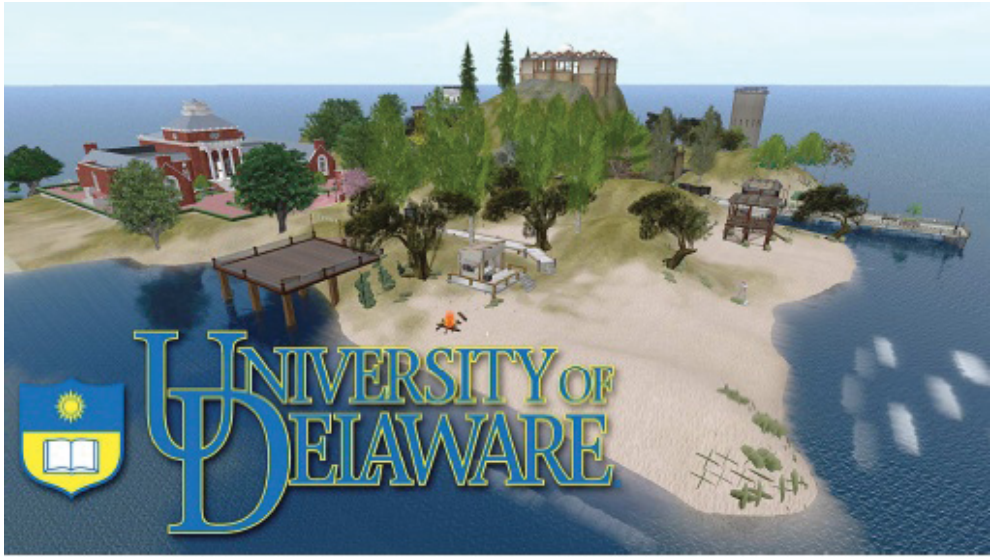

Figure 8: University of Delaware's first island with flexible teaching spaces [22].

There are other details about SL, which make it into an appropriate platform in terms of environmental simulations. For example SL has its own currency, which called Linden Dollar (L\$). It has its own day/night length. Traditionally, "Days" are 3 hours long, and "Nights" are 1 hour long, but this setting can be overridden by private estate owners. For example, users can stop the sun cycle, or speed it up, or slow it down [23].

Many studies researched SL potentials for education [24], health related activities $[25,26]$ and other fields. As a result, it seems that SL (in specific and other VEs in general) looks like a good candidate as a platform to simulation purposes. These simulations can help us to understand how environmental phenomena will evolve through time and how can they affect the earth nature during their evolution process.

Practically, it is impossible to employ SL for a realistic future identification (As a fundamental goal of RD); unless by benefiting from sophisticated algorithms with higher levels of flexibility and precision. Therefore, GA is considered as a practical tool to determine more realistic options for future artificial environments. Such algorithms are being developed on the basis of the history data of each economic, social and environmental flow.

\section{Discussion}

As a short communication, this paper has introduced the RD and its principal intentions, such as higher consideration of the inter-relationship between flows, and rebirth-rebuilding-and recover concept. Moreover, the paper has highlighted the role of providing precision in future identification, in preventation of the unlikely wastes of recourses during the processes of infrastructure over-design over the present and future systems. Such over-designs are being carried out to deal with risks and future changes.

To continue, the GA has been introduced as one of the most effective approaches to develop more precise Regenerative Evolutionary Strategies. In that context, the GA has been considered as a tool to simulate the possibilities of change, over the flows' interactions in-with the proposed models. Thus, the GA proposes the optimum solutions to address the human demands and deal with all flows of socio-economic and environmental changes, while the constraints are considered. Such solutions are strongly suggested to be taken into account during the propositions of the Virtual Environments, to be designed to examine the possibilities of future universes (refer to section 4). This action in turn, is suggested as a more comprehensive approach to design such virtual systems with more realistic and practical results. These results will practically cause more efficient Regenerative Design and Decisionmaking strategies, and will save huge amounts of energy, efforts, and natural resources, through longer systems' time-frames.

\section{Conclusion}

In order to develop an effective EAS, the following points must be considered:

- To develop more precise algorithms, to quantify the evolution of socio-economic and environmental flows; 
Citation: Kashkooli AMS, Daneshi E, Hashemian AM (2015) A Critical Approach for Evolution Analysis in Regenerative Developments. Int J Swarm Intel Evol Comput 4: 119. doi: 10.4172/2090-4908.1000119

- To take advantage from virtual environments to practically examine the future possibilities in advance, before any decisionmaking for over-design of the systems' infrastructures.

This paper opens doors to the future researches and practices in the following terms: Regenerative software developments; development of precise evolution analysis systems; and over-design management.

\section{References}

1. Cole RJ(2011) Transitioning from green to regenerative design. Building Research \& Information 40: 39-53

2. Cole RJ (2011) Regenerative design and development: current theory and practice. Building Research \& Information 40: 1-6

3. Lewis S (2010) Energy, Climate and Politics.

4. Cole $\mathrm{RJ}(2011)$ A regenerative design framework: setting new aspirations and initiating new discussions. Building Research \& Information 40: 95-111

5. Cole RJ and Kashkooli AMS( 2013) Clarifying Net Positive Energy, in Stream 5 - Pushing the Boundaries: Net Positive Buildings (SB13), CaGBC National Conference and Expo.: Vancouver, BC, Canada.

6. Livingston P and Stark J ( 2013) Lecture: Transition Towns - A Localized Planetarian Response.

7. RLI (2013) What is regenerative design?

8. Computing-For-Sustainability.Trajectory of environmentally responsibledesign (2010)

9. Rigosi A and Rueda FJ (2012) Propagation of uncertainty in ecological models of reservoirs: From physical to population dynamic predictions. Ecological Modelling. 247: 199-209

10. Kolokotsa $\mathrm{D}(2005)$ Implementation of an integrated indoor environment and energy management system. Energy and Buildings. 37: 93-99

11. Kashkooli AMS (2013) A Road Map Towards Sustainable Building Design: State of Art in Realistic Decision Making., Germany: LAP Lambert Academic Publishing.

12. Back T, Fogel DB, Michalewicz Z ( 1997) Handbook of Evolutionary Computation: IOP Publ. Ltd., Bristol, UK.
13. Kakas AC ( 2011) Ant Colony Optimization, in Encyclopedia of Machine Learning 36-39

14. Hollan JH ( 1992) Adaptation in Natural and Artificial Systems: An Introductory Analysis with Applications to Biology, Control, and Artificial Intelligence. The Quarterly Review of Biology 69

15. Pham DT (2011) The Bees Algorithm-A Novel Tool for Complex Optimisation in Intelligent Production Machines and Systems-2nd I* PROMS Virtual International Conference 3-14 July

16. Gandomi AH, Yang XS, Alavi AH (2013) Cuckoo search algorithm: A metaheuristic approach to solve structural optimization problems. Engineering with Computers. 29: 17-35

17. Fonseca CM and Fleming PJ (1993) Genetic Algorithms for Multiobjective Optimization: FormulationDiscussion and Generalization. International Computer Games Association (ICGA) 93: 416-423

18. Fonseca CM and Fleming PJ (1998) Multiobjective optimization and multiple constraint handling with evolutionary algorithms - Part I: A unified formulation. IEEE Transactions on Systems, Man, and Cybernetics Part A:Systems and Humans 28: 26-37

19. Linden-Lab.( 2013 ) Infographic: 10 Years of Second Life.

20. Ducheneaut N(2009) Body and Mind: A Study of Avatar Personalization in Three Virtual Worlds.in CHI, Massachusetts, USA.

21. Erickson T (2011) Synchronous Interaction Among Hundreds: An Evaluation of a Conference in an Avatar-based Virtual Environment. in $\mathrm{CHI}$,Vancouver, BC, Canada

22. Jeffers D (2008) Is There a Second Life in Your Future?, in SIGUCCS'08: Portland, Oregon, USA.

23. Second-Life-CommunityLand.(2009)

24. Warburton S. (2009) Second Life in higher education: Assessing the potentia for and the arriers to deploying virtual worlds in learning and teaching. British Journal of Educational Technology 40: 414-426

25. Beard L ( 2009) A Survey of Health-Related Activities on Second Life. Journal of medical Internet research.

26. Boulos MNK, Hetherington L†Wheeler S (2007) Second Life: an overview of the potential of $3-D$ virtual worlds in medical and health education. Health Information and Libraries Journal. 24: 233-245 